PREPARED FOR THE U.S. DEPARTMENT OF ENERGY, UNDER CONTRACT DE-AC02-76CH03073

PPPL-3830

PPPL-3830

UC-70

\title{
Particle-in-cell Simulations \\ of Raman Laser Amplification in Preformed Plasmas
}

by

Daniel S. Clark and Nathaniel J. Fisch

June 2003

N/M|

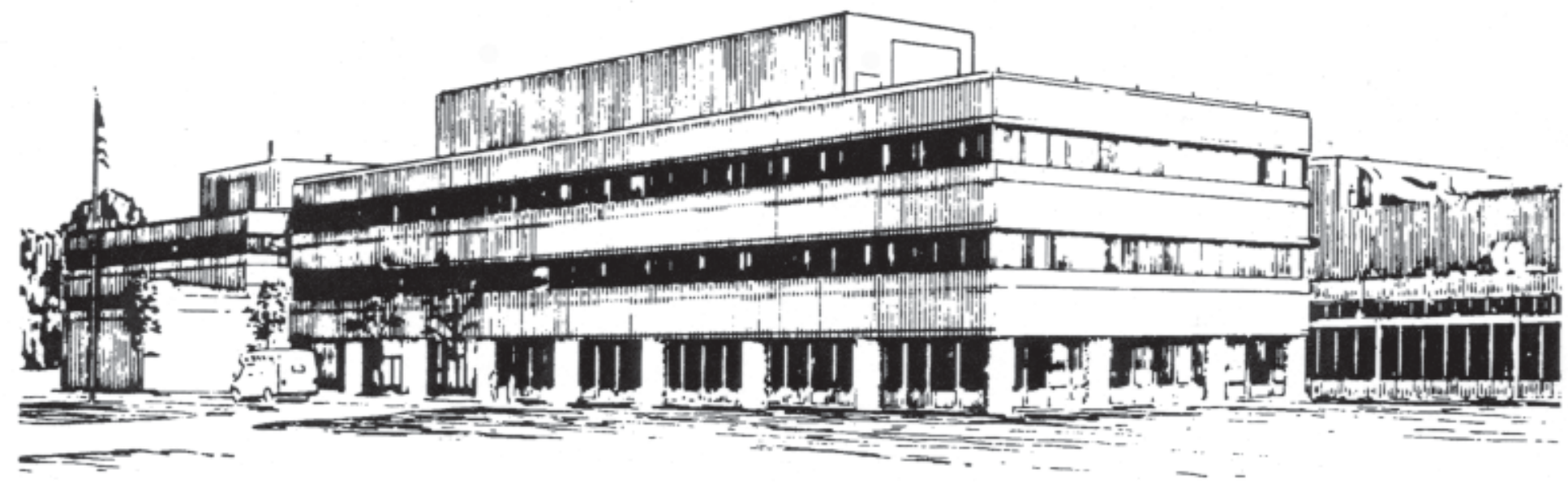

PRINCETON PLASMA PHYSICS LABORATORY PRINCETON UNIVERSITY, PRINCETON, NEW JERSEY 


\section{PPPL Reports Disclaimer}

This report was prepared as an account of work sponsored by an agency of the United States Government. Neither the United States Government nor any agency thereof, nor any of their employees, makes any warranty, express or implied, or assumes any legal liability or responsibility for the accuracy, completeness, or usefulness of any information, apparatus, product, or process disclosed, or represents that its use would not infringe privately owned rights. Reference herein to any specific commercial product, process, or service by trade name, trademark, manufacturer, or otherwise, does not necessarily constitute or imply its endorsement, recommendation, or favoring by the United States Government or any agency thereof. The views and opinions of authors expressed herein do not necessarily state or reflect those of the United States Government or any agency thereof.

\section{Availability}

This report is posted on the U.S. Department of Energy's Princeton Plasma Physics Laboratory Publications and Reports web site in Fiscal Year 2003. The home page for PPPL Reports and Publications is: http://www.pppl.gov/pub_report/

DOE and DOE Contractors can obtain copies of this report from:

U.S. Department of Energy

Office of Scientific and Technical Information

DOE Technical Information Services (DTIS)

P.O. Box 62

Oak Ridge, TN 37831

Telephone: (865) 576-8401

Fax: (865) 576-5728

Email: reports@adonis.osti.gov

This report is available to the general public from:

National Technical Information Service

U.S. Department of Commerce

5285 Port Royal Road

Springfield, VA 22161

Telephone: $1-800-553-6847$ or

(703) $605-6000$

Fax: (703) 321-8547

Internet: http://www.ntis.gov/ordering.htm 


\title{
Particle-in-cell simulations of Raman laser amplification in preformed plasmas
}

\author{
Daniel S. Clark* and Nathaniel J. Fisch ${ }^{\dagger}$ \\ Plasma Physics Laboratory, Princeton University, P.O. Box 451, Princeton, New Jersey 08543
}

(Dated: June 27, 2003)

\begin{abstract}
Two critical issues in the amplification of laser pulses by backward Raman scattering in plasma slabs are the saturation mechanism of the amplification effect (which determines the maximum attainable output intensity of a Raman amplifier) and the optimal plasma density for amplification. Previous investigations [V. M. Malkin, et al. , Phys. Rev. Lett., 82(22):4448-4451, 1999] identified forward Raman scattering and modulational instabilities of the amplifying seed as the likely saturation mechanisms and lead to an estimated unfocused output intensities of $10^{17} \mathrm{~W} / \mathrm{cm}^{2}$. The optimal density for amplification is determined by the competing constraints of minimizing the plasma density so as to minimize the growth rate of the instabilities leading to saturation but also maintaining the plasma sufficiently dense that the driven Langmuir wave responsible for backscattering does not break prematurely. Here, particle-in-cell code simulations are presented which verify that saturation of backward Raman amplification does occur at intensities of $\sim 10^{17} \mathrm{~W} / \mathrm{cm}^{2}$ by forward Raman scattering and modulational instabilities. The optimal density for amplification in a plasma with the representative temperature of $T_{e}=200 \mathrm{eV}$ is also shown in these simulations to be intermediate between the cold plasma wave-breaking density and the density limit found by assuming a water bag electron distribution function.
\end{abstract}

\section{INTRODUCTION}

To overcome the diffraction grating damage thresholds which limit the peak laser intensities achievable by the the Chirped Pulse Amplification (CPA) technique [1, 2], the backward Raman amplification scheme has recently been proposed in which a plasma is used as the laser amplifying medium [3]. In this scheme, a long pumping laser pulse is collided with a short seed pulse down-shifted in frequency from the pump by the plasma frequency in a preformed plasma slab. The seed pulse serves to stimulate the Raman backscatter of the pump into the seed with the result that, in the nonlinear regime, a characteristic $\pi$-pulse forms from the seed which both amplifies and compresses linearly with time. Theoretically, unfocused intensities of $10^{17} \mathrm{~W} / \mathrm{cm}^{2}$ in $\sim 50 \mathrm{fs}$ pulses are accessible by this technique for $1.064 \mu \mathrm{m}$-radiation. By focusing such pulses to near the diffraction limit, intensities of $\sim 10^{25} \mathrm{~W} / \mathrm{cm}^{2}$ become conceivable - a value $10^{4-5}$ times greater than achievable by current CPA techniques. At shorter wavelengths, even higher intensities may be feasible. Moreover, backward Raman amplification schemes envision the combination of two optical systems: one optical system consisting of one or several pump beams delivering large powers over a wide aperture with low requirements on optical precision, and a second system to deliver a higher precision but lower power and smaller aperture seed pulse to extract and focus the pump energy. Hence, by separating the high power and high precision optical systems, a likely more robust, as well as higher power, amplifier might be realized [4].

*Electronic address: dclark@pppl.gov

${ }^{\dagger}$ Electronic address: fisch@pppl.gov
Analogous to the nonlinear distortions and thermal degradations which limit output intensities for schemes based on solid material amplifiers or gratings (such as the CPA technique), Raman amplification is limited (at much higher intensities) by instabilities which can arise from the interaction of the intense light pulses with the plasma. That is, energy may be transfered from the pump to the seed and the seed pulse amplified only for as long as the growth time of the fastest growing instability. After that point, the plasma must be terminated, and the amplified seed directed to its target before it is broken apart by the developing instability. For the Raman amplification regime, forward Raman scattering (FRS) [5] and modulational [6-8] instabilities of the ultra-intense amplifying seed are the most likely saturation mechanisms. The growth times for these instabilities, and hence the amount of amplification of the seed, may be maximized by minimizing the background plasma density. Since the exchange of energy between the pump and seed by Raman scattering is mediated by a Langmuir wave whose amplitude (in the nonlinear regime) is essentially determined by the strength of the pump, the plasma density for a given pump intensity is limited from below by the threshold at which the driven Langmuir wave would begin to break and interfere with the backscattering of the pump. Optimal performance of a Raman amplifier is then attained at the threshold for wave-breaking with a plasma whose length is of the order of the minimum of the growth lengths of the FRS or modulational instabilities of the seed. Coincidentally, at the wave-breaking threshold, these growth lengths are approximately equal. In principle, the amplified seed pulse is also unstable to backward Raman scattering (BRS). Given that the seed pulse is compressing to $\sim 50$ fs pulse widths, any backscattered signal is, however, rapidly convected out of the pulse, and the growth of this instability should be very limited. 
Previous investigations of backward Raman amplification have largely utilized a cold plasma, 3-wave description of the resonant Raman interaction [3, 9-11] where optimal amplification was assumed to occur close to the cold plasma wave breaking threshold and that the amplification effect would saturate after approximately one growth time of the FRS or modulational instabilities. The numerical models employed by these studies, however, did not include the physics of wave-breaking or saturation. Some modeling with the particle-in-cell (PIC) code XOOPIC [12] has also been performed [13], in particular to investigate amplification in plasma channels [14], but there has been no systematic application of the fully kinetic model of the plasma provided by a PIC code to the problem of saturation of Raman amplification or the details of wave-breaking in amplification. Further, given that the pump beam in a Raman amplifier can be expected to heat the plasma to $\sim 100 \mathrm{eV}$ by the time the seed pulse is injected into the plasma and also based on considerations of the stability of the pump beam to backscatter from thermal plasma noise [15], the effect of wave-breaking on amplification in a finite temperature plasma is of particular interest. This paper specifically investigates these issues of saturation and wave-breaking in amplification for a plasma with the representative temperature of $T_{e}=200 \mathrm{eV}$ using the PIC code Zohar [16].

This paper is organized as follows. Sec. II briefly reviews the phenomenology of Raman amplification and the numerical modeling techniques as will be needed in the subsequent investigations of wave-breaking and saturation. Sec. III investigates the optimal density for Raman amplification in a plasma of $T_{e}=200 \mathrm{eV}$ from the perspective of breaking of the backscatter-driven Langmuir wave. Sec. IV then investigates the saturation of Raman amplification at this optimal density, and Sec. V summarizes and concludes.

\section{PHENOMENOLOGY OF RAMAN AMPLIFICATION}

To fix the concepts and terminology of Raman amplification, a canonical example of the amplification process is shown in Fig. 1. The pump (shown in blue) is propagated across the preformed plasma from $x=0$ to $x=8,000 c / \omega_{1}$. When the pump has reached the far side of the plasma, a seed (shown in green) with a "flattopped" Gaussian initial shape and amplitude equal to that of the pump is launched in the opposite direction. The interaction of the pump and seed pulses generates a Langmuir wave (shown in red) which serves to backscatter the pump into the seed. Quickly, for these parameters, the seed amplifies and fully depletes the pump. After a very brief phase of broadening and exponential amplification (up to approximately $\omega_{1} t \simeq 10^{4}$ ), the seed begins to narrow and amplify linearly in time concomitant with pump depletion. By $\omega_{1} t=1.3 \times 10^{4}$, the multipeaked, characteristic structure of the $\pi$-pulse shape of



FIG. 1: Example 3-wave simulation of Raman amplification with $a_{1}=a_{2}(t=0)=0.006, n_{e}=0.1 n_{c}, T_{e}=200 \mathrm{eV}$, and linear polarization.

the amplified seed is evident, and by $\omega_{1} t=1.56 \times 10^{4}$ amplification by a factor of $\sim 100$ in intensity has been accomplished. Given the low damping rates and weak dispersion for these plasma parameters, the Langmuir wave persists throughout the plasma in the wake of the seed pulse.

This example was computed using the most elementary 3-wave description of the Raman interaction similar to that described in Ref. [3] where only the resonant pump and seed laser envelopes and the envelope of the resonant Langmuir wave are followed. Here, $a_{1,2} \doteq|e|\left\langle A_{1,2}\right\rangle / m_{e} c^{2}$ are the slowly-evolving normalized envelopes of the pump and seed laser vector potentials and $a_{3} \doteq|e|\left\langle E_{3}\right\rangle / m_{e} c \sqrt{\omega_{1} \omega_{p e}}$ is the normalized envelope of the resonant Langmuir wave amplitude. Analogously, $\omega_{1}$ and $\omega_{2}$ denoted the pump and seed laser frequencies, respectively, which are mismatched by the plasma frequency $\omega_{p e}=\omega_{1}-\omega_{2}$ (neglecting thermal effects). In the following, only linearly polarized pump and seed lasers are considered, though the results for circularly polarized lasers would be similar.

Note that, for the sake of illustrating rapid amplification into the nonlinear regime and a clearly formed $\pi$-pulse, the plasma density has been chosen to be $n_{e}=$ $0.1 n_{c}$ which is well above the wave-breaking limit considered optimal for Raman amplification. The temperature of $T_{e}=200 \mathrm{eV}$ used here and below is again motivated from seeking consistency with the constraints due to backscatter from thermal noise [15] and additionally that the pump beam can be expected to heat the preformed plasma to $\sim 100 \mathrm{eV}$ by the time the seed enters.

As a first step in verifying the expectations for Raman amplification based on the 3-wave model illustrated above, the more rigorous and detailed PIC model of the plasma can be applied to a simulation of Raman amplification for the same parameters used in Fig. 1. However, 
as is well-known, PIC codes support far higher electrostatic fluctuation levels than should be expected for a physical weakly-coupled plasma [17]. These enhanced fluctuations are essentially the result of having far fewer particles per Debye sphere in any reasonable simulation than would be present in a physical plasma resulting in very large statistical fluctuations in the plasma properties. Physically, this is equivalent to an artificially high thermal Langmuir wave fluctuation level which (like the injected seed pulse in a Raman amplifier) can serve to seed the Raman backscatter of the pump. Given the very rapid growth rate for Raman backscattering, these exaggerated fluctuation levels can very rapidly deplete the pump and completely suppress the intended amplification of the seed. Directly repeating the simulation shown in Fig. 1 with Zohar illustrates precisely this effect: Before the seed has even been injected into the plasma, strong backscattering from the exaggerated Langmuir fluctuations has almost completely depleted the pump and very limited amplification of the seed ultimately results. Increasing the number of particles can reduce the background Langmuir fluctuation level accounting for this depletion. It might then be hoped that a PIC simulation of Raman amplification which was stable to Langmuir noise could serve as a worst-case proof of the viability of Raman amplification in the face of the much lower noise levels to be expected in a physical plasma. Unfortunately, such a simulation could not be accomplished with a reasonable number of particles and a plasma length comparable to that of interest for an amplifier.

As originally proposed in Ref. [18], linear backscattering from noise can be suppressed while useful nonlinear Raman amplification of the seed persists if the Raman interaction is appropriately detuned (e.g., by introducing a density gradient or chirp of the pump beam). This stabilization of the amplifier or "nonlinear filtering" of the amplification effect relies on the narrowing of the seed with its amplification during the nonlinear phase. Since the seed is rapidly narrowing in space during its nonlinear amplification, its consequent broadening in frequency renders this nonlinear amplification phase robust to any "detuning" from the exact Raman resonance. The linear evolution of the premature backscattering of the pump (a narrow band interaction in frequency as evidenced by the initial spatial broadening of any signal), however, would not be resilient to such deviations from resonance. The introduction of a spatial variation in the plasma frequency (i.e., a density gradient) or a temporal variation in the pump frequency (a frequency chirp representing a spatio-temporal variation in the pump frequency) can then be used to detune or suppress the linear pump backscatter from noise without disrupting the intended nonlinear pump backscatter from the seed. Combining a density gradient and frequency chirp may be particularly useful in that a selective detuning of the FRS instability of the amplifying seed may be possible [19]. If the pump were chirped in the opposite sense to any density gradient detuning, then the detuning experienced by the intended
Raman backscatter of the pump would be only the difference of the detunings due to the density gradient and the chirp. The FRS of the seed, however, (which occurs independently of the pump) would experience the stronger detuning due to the density gradient alone. A selecting or emphasizing of the intended backscatter effect is then possible.

Nevertheless, again on account of the exaggerated fluctuation levels present in the PIC code, stabilization of the pump to thermal backscatter in Zohar simulations could only be accomplished by introducing an unreasonably steep density gradient. In principle, as discussed by [18], the detuning due to a large density gradient can be overcome and a $\pi$-pulse attractor formed provided that the initial seed pulse is sufficiently intense. That is, the seed must reach the nonlinear regime (by depleting the pump) and be compressed sufficiently rapidly that its bandwidth in this regime is greater that the detuning which it experiences in propagating through the density gradient. A series of simulations might then be run to determine the initial seed amplitude or bandwidth needed to maintain the Raman resonance in a plasma with a density gradient sufficiently steep to control premature backscatter from noise. However, as emphasized above, these results would only describe criteria for stable Raman amplification in these model simulation plasmas with their extreme and unphysical noise levels. Regarding physically realizable plasmas with their almost certainly much lower fluctuations levels, such results would be inapplicable.

However, the option exists in a one dimensional PIC code artificially to suppress Raman backscatter in regions of the plasma ahead of the seed pulse. This can easily be accomplished by simply "switching off" the $\mathbf{v} \times \mathbf{B}$ force (and hence the ponderomotive force driving the Raman instability) for all particles slightly to the left of the seed pulse front. The result of such a run using the same parameters as Fig. 1 is shown in Fig. 2. Comparing with

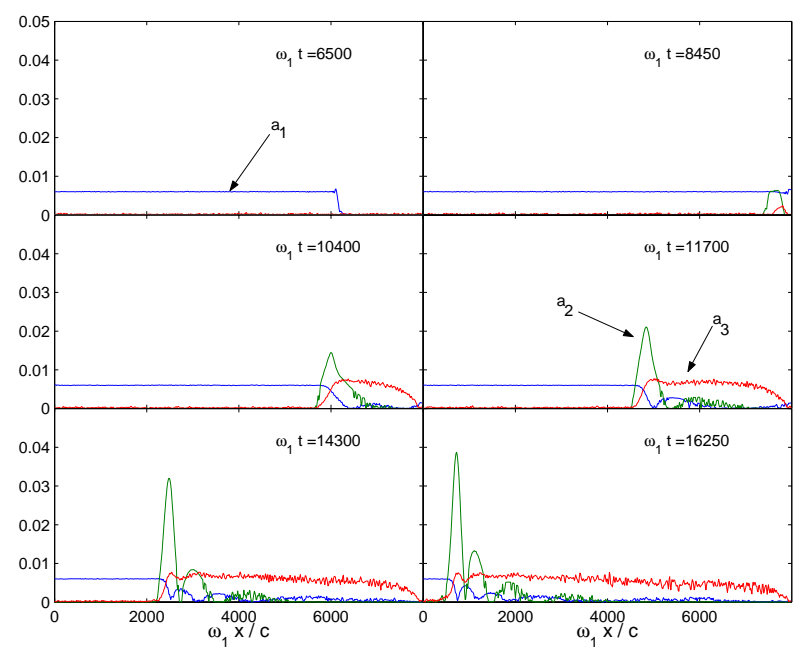

FIG. 2: Example Zohar simulation of Raman amplification for the same parameters as in Fig. 1. Raman backscatter has been artificially suppressed ahead of the seed pulse front. 
Fig. 1, this run serves to verify kinetically the underlying validity of the 3 -wave description for amplification into the nonlinear, $\pi$-pulse regime. A slightly lesser degree of peak seed amplification is seen in the PIC run, but a $\pi$-pulse is clearly seen to form with the appropriate degree of pump depletion and appropriate Langmuir wave profile. This result was found independent of the exact location at which the $\mathbf{v} \times \mathbf{B}$ force was switched on (provided premature pump depletion was suppressed) indicating that this modification of the plasma properties does not alter the amplification dynamics.

\section{EFFECT OF LANGMUIR WAVE-BREAKING}

Having verified the formation of the basic $\pi$-pulse in Raman amplification using the detailed PIC code model of the plasma, this section addresses the modifications to Raman amplification which result from breaking of the backscatter-driven Langmuir wave. As shown in Ref. [3], specifying a certain pump intensity and wavelength (or equivalently $a_{1}$ ) specifies the length of the amplifier (approximately one growth length of the FRS or modulational instabilities) and the optimal plasma density for amplification (the density at which the driven Langmuir wave should just break). For linear polarization, these parameters are given by $L_{\mathrm{amp}} \sim c / \omega_{1} a_{1}^{2}$ and $n_{e}=n_{c}\left(2 \sqrt{2} a_{1}\right)^{4 / 3}$. Choosing $a_{1}=0.015$ so as to adjust the optimal amplification length to match the reasonable simulation lengths shown in Fig. 2, namely $\omega_{1} L_{\mathrm{amp}} / c=8,000$, the threshold density for breaking of the backscatter-driven Langmuir wave is then $n_{e} \simeq$ $0.015 n_{c}$. Using the same method of noise suppression as Fig. 2, Fig. 3 shows the results of a Zohar simulation at this cold plasma wave-breaking limit. The Langmuir

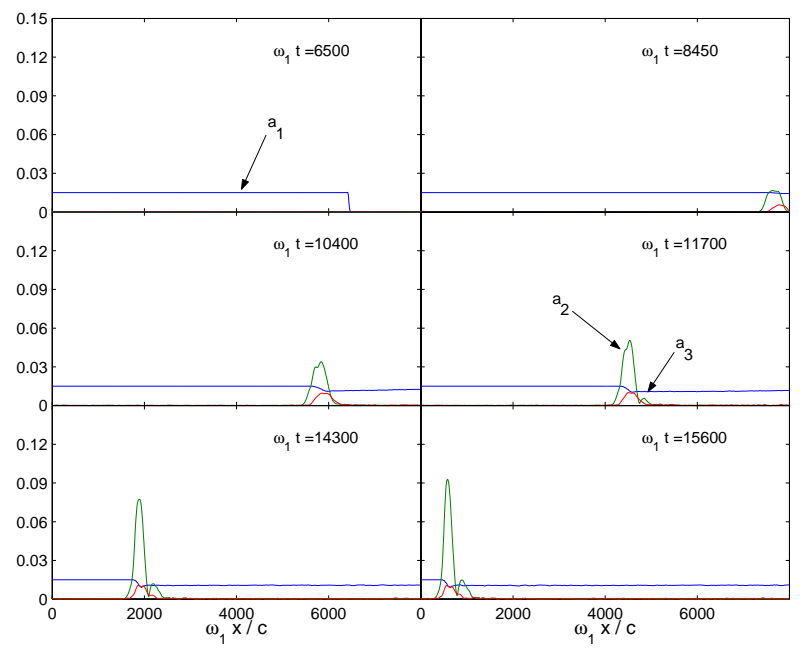

FIG. 3: Zohar simulation of Raman amplification at the cold plasma wave-breaking limit with $a_{1}=a_{2}(t=0)=0.015$, $n_{e}=0.015 n_{c}, T_{e}=200 \mathrm{eV}$, and linear polarization. wave is seen to break in the neighborhood of the maximum of the seed pulse allowing only $\sim 30 \%$ depletion of the pump. Interestingly, even after the point at which the Langmuir wave breaks, a small secondary oscillation in the seed pulse (suggestive of the oscillations of the fully formed $\pi$-pulse) is evident.

The relatively low depletion of the pump in this simulation is evidence of the effect of the finite plasma temperature. Using the approximation of a "water bag" model for the electron distribution function, Ref. [20] showed that thermal effects reduce the electric field at which wave-breaking can be expected by a factor of $\left(1+2 \beta^{1 / 2}-8 \beta^{1 / 4} / 3-\beta / 3\right)^{1 / 2}$ from the cold plasma value [21] with $\beta \doteq 3 v_{\text {te }}^{2} / v_{\text {phase }}^{2}$. Physically, allowing for a finite plasma temperature implies a small population of energetic electrons close to the phase velocity of the electrostatic wave. Such electrons can more easily be swept into resonance with the wave than in the case of a distribution with zero temperature and hence lead to a lower threshold at which the wave will nonlinearly break. For the $T_{e}=200 \mathrm{eV}$ initialized in the simulation, accounting for thermal effects by this "water bag" model raises the breaking threshold to $n_{e} \simeq 0.05 n_{c}$, i.e., a factor of three greater than the cold plasma threshold of $n_{e} \simeq 0.015 n_{c}$. That the Langmuir wave is well into the wave-breaking regime is confirmed by the electron phase space snapshot for $\omega_{1} t=15,600$ shown in Fig. 4 . The substantial ac-

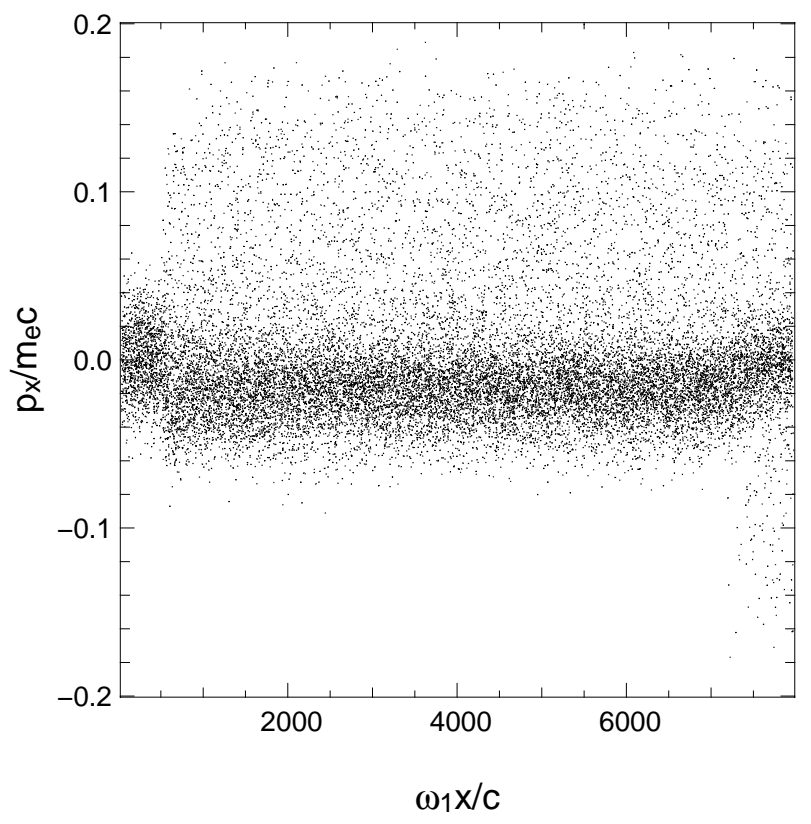

FIG. 4: $x-p_{x}$ electron phase space from the simulation shown in Fig. 3 for $\omega_{1} t=15,600$. Strong asymmetric heating of the electron distribution corresponds to breaking of the backscatter-driven Langmuir wave.

celeration of the electron population in the direction of the Langmuir wave phase velocity behind the seed pulse (typically described as a flattening of the distribution) corresponds to electrons from the initially Maxwellian 
distribution being rapidly pulled into resonance with the wave. Balancing this rapid energy gain by the electrons, a strong and abrupt damping of the Langmuir wave clearly results.

Greater depletion of the pump can be accomplished by increasing the plasma density, as in the simulation shown in Fig. 5 with $n_{e}=0.025 n_{c}$. Similar to the initial

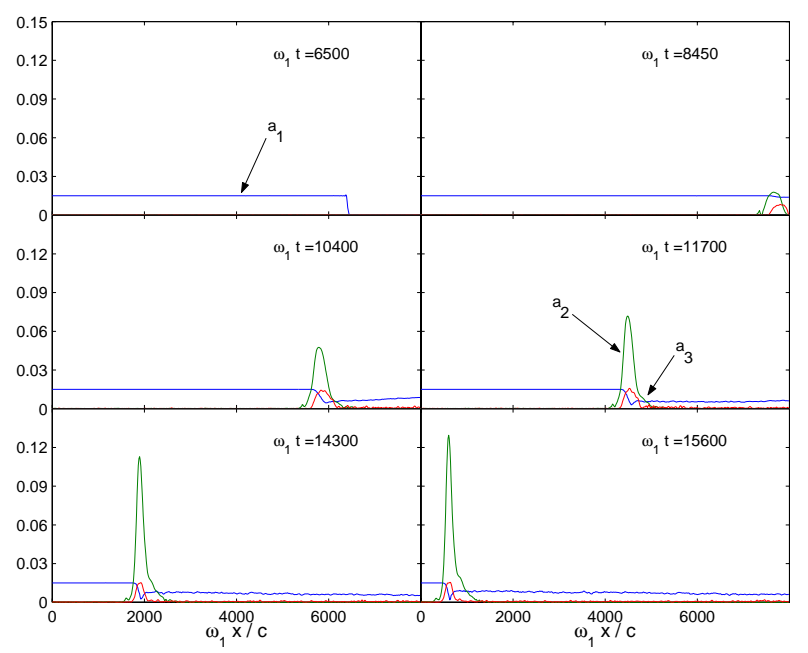

FIG. 5: Zohar simulation of Raman amplification above the cold plasma wave-breaking limit. The simulation parameters are the same as in Fig. 3 but with $n_{e}=0.025 n_{c}$.

results of Ref. [3], close to the wave-breaking threshold a single spike forms for the amplifying pulse, i.e., the secondary oscillations of the $\pi$-pulse are completely suppressed. This suppression results, again, from the breaking of the Langmuir wave near the peak of the seed pulse and subsequent disruption of the $\pi$-pulse solution behind this point. Stronger depletion of the pump and hence stronger amplification of the seed are evident than in Fig. 3. Note that, though the pump is more strongly depleted at this density than in Fig. 3, the breaking of the Langmuir wave still results in less depletion than for a fully formed $\pi$-pulse as, for example, in Fig. 2.

Still greater depletion of the pump can be achieved by increasing the plasma density further as shown in the simulation results in Fig. 6 with $n_{e}=0.035 n_{c}$. However, this extra energy extraction from the pump appears to result only in the formation of the secondary oscillations of a partial $\pi$-pulse but does not increase the peak amplification of the leading spike. Breaking of the Langmuir wave is still evident (as can be verified from a phase space plot similar to Fig. 4) but is not as abrupt as in the lower density cases.

In contrast to the expectations for a cold plasma, Figs. 5 and 6 indicate that, for finite temperatures, optimal amplification occurs for a plasma density intermediate between the cold plasma breaking threshold $\left(n_{e} \simeq 0.015 n_{c}\right)$ and the threshold given by the formula of [20] $\left(n_{e} \simeq 0.05 n_{c}\right)$. For these parameters, $n_{e} \simeq 0.025 n_{c}$ yields the single optimally amplified spike, while below



FIG. 6: Zohar simulation of Raman amplification above the cold plasma wave-breaking limit. The simulation parameters are the same as in Fig. 3 but with $n_{e}=0.035 n_{c}$.

and above this density less amplification or amplification of only the tail of the pulse results. Simultaneously, increasing $n_{e}$ to greater than $0.025 n_{c}$ can be expected to accelerate the onset of the FRS or modulational instabilities resulting in less net amplification. Hence, $0.025 n_{c}$ can be considered the optimal density for amplification.

As can be expected, repeating the simulations in Figs. 3-6 with initial temperatures of $10 \mathrm{eV}$ shows that optimal amplification occurs for $n_{e} \simeq 0.015 n_{c}$ in agreement the cold plasma prediction. Again, due to considerations of backscatter from noise as well as the heating of the plasma by the pump beam prior to the injection of the seed, such low plasma temperatures are untenable for a realistic Raman amplifier. That the thermal modifications to the wave-breaking threshold were calculated using an approximate "water bag" model of the electron distribution function should be borne in mind here. Interestingly, by inspecting the $x-p_{x}$ phase space for these simulations, evidence of the effects of strong electron acceleration in the direction of the Langmuir wave phase velocity after the passage of the seed pulse (i.e., breaking of the Langmuir wave) persists until the density is greater than $\sim 0.1 n_{c}$, a value approximately seven times greater than the threshold expected for a cold plasma.

\section{SATURATION OF AMPLIFICATION}

Despite the simulation lengths for Figs. 5 and 6 being adjusted to match the growth lengths for the FRS and modulational instabilities of amplifying seed, in neither of these simulations is any sign of saturation of the amplification evident. In part, this is the result of the one dimensional nature of these simulations. Specifically, the gain rate for FRS in one dimension (i.e., directly forward) is a factor of $\sqrt{\omega_{p e} / \omega_{2}}$ smaller than the gain rate 
in two or more dimensions (i.e., near forward scatter) $[22,23]$ as used in the output estimates of Ref [3]. For these parameters, this is a reduction by a factor of $\sim 3$. The modulational instability is not affected by this distinction. More generally, the estimated amplifier length cited in Sec. II corresponds approximately to only one exponentiation of the FRS or modulational instabilities at the threshold density for wave-breaking. The stable amplifier length is more precisely the product of one gain length with the logarithm of the number of exponentiations tolerable by the seed from the noise source present for this instability.

Without addressing the details of the noise source for either of these instabilities, saturation may be studied simply by increasing the length of the simulation domain. Figs. 7 and 10 show the result of repeating the simulations of Figs. 5 and 6 with the simulation size increased by a factor of four. Between $\omega_{1} t=57,200$ and $\omega_{1} t=62,400$

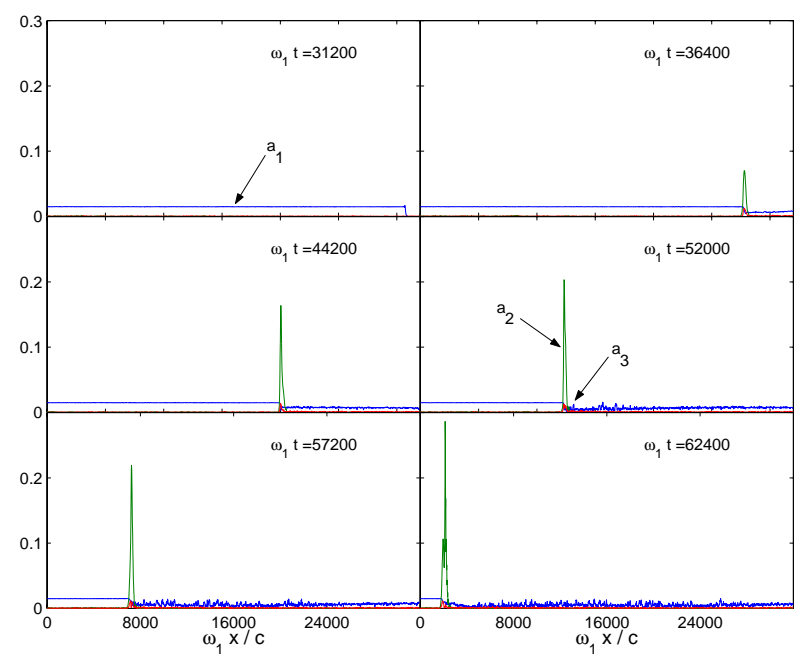

FIG. 7: Zohar simulation showing saturation of Raman amplification near the wave-breaking limit: $n_{e}=0.025 n_{c}$. The simulation parameters are the same as in Fig. 5 but with the simulation domain lengthened by a factor of four.

in Fig. 7, the amplification effect has clearly been saturated and the seed pulse has begun to broaden and break apart. The result of zooming in on the seed pulse in the last to frames of Fig. 7 is shown in Fig. 8. A strong spike has formed at the tail of the seed pulse which, though in principle is an amplification of the seed, indicates the disruption of the $\pi$-pulse and the termination of the contracting and amplifying self-similar solution. Just prior to saturating, the single amplified spike has reached an amplitude of $a_{2} \sim 0.25$ or intensity of $3.4 \times 10^{17} \mathrm{~W} / \mathrm{cm}^{2}$ for $\lambda=0.532 \mu \mathrm{m}$ in quite close agreement with the value expected from Ref. [3].

The nonlinear mechanism responsible for this saturation can be identified from the $k_{x}$ spectrum for this simulation shown in Fig. 9. Here, the power spectrum of rightpropagating energy (i.e., in the direction of the pump) is shown in blue, the spectrum of left-propagating energy

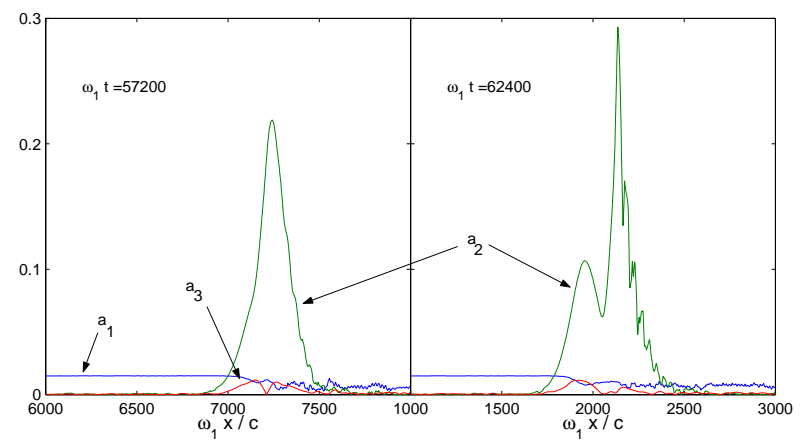

FIG. 8: Result of zooming in on the seed pulses from the last two frames of Fig. 7. The $\pi$-pulse shape breaks apart indicating saturation of the amplification mechanism.

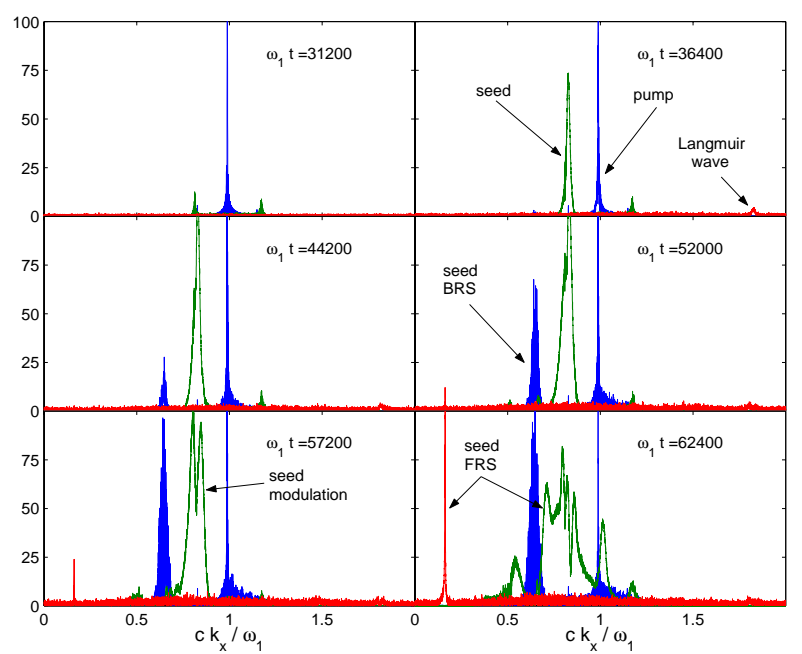

FIG. 9: $k_{x}$ spectrum from the Zohar simulation of Fig. 7. The right-propagating power spectrum is shown in blue, the left-propagating spectrum in green, and the spectrum of the longitudinal electric field is shown in red.

(in the direction of the seed) is shown in green, and the spectrum of the longitudinal electric field (i.e., the Langmuir wave) is shown in red. Initially, the seed pulse is seen to amplify as well as broaden in $k_{x}$ corresponding to its spatial narrowing with amplification. The resonantly driven Langmuir wave corresponds to the small signal at $c k_{x} / \omega_{1} \simeq 1.84$. The forking of the seed pulse signal evident for $\omega_{1} t=57,200$ and substantial broadening of the seed spectrum for $\omega_{1} t=62,400$ without any accompanying signal in the electrostatic field suggests saturation by the modulational instability. A small degree of Raman forward scattering of the seed is apparent for $\omega_{1} t>57,200$ as the right propagating signal at approximately the same $k_{x}$ as the seed BRS signal and the substantial Langmuir oscillation at $k_{x} \simeq \omega_{p e} / c \simeq 0.16 \omega_{1} / c$. Again, since only directly forward or backward scattering is possible in this one dimensional simulation, the gain rate for FRS is significantly reduced from the three dimensional value (near forward) cited above and from the 
gain rate of the modulational instability.

Remarkably, a substantial degree of Raman backscatter of the seed pulse is seen from $\omega_{1} t=44,200$ onwards in the form of the right-propagating signal down-shifted from the seed pulse signal by $\omega_{p e} / c$. This instability is evident in $x$-space as the chaotic oscillations in the pump pulse after the seed has passed for $\omega_{1} t>52,000$. Given the narrowing of the seed with its amplification, this scattering instability is expected to have a fixed and limited convective gain. In the case of this simulation, however, the very high and broad-band Langmuir noise source (as evidenced in Fig. 9) represents a strong enough seed for this instability to develop substantially.

In the case of Fig. 10, the higher plasma density predictably results in the more rapid onset of the modulational instability and saturation of the seed amplification (occuring for $\omega_{1} t \sim 5 \times 10^{4}$ as compared to $\omega_{1} t \sim 6 \times 10^{4}$ in Fig. 7). Note that, while not evident in comparing



FIG. 10: Zohar simulation showing saturation of Raman amplification near the wave-breaking limit: $n_{e}=0.035 n_{c}$. The simulation parameters are the same as in Fig. 6 but with the simulation domain lengthened by a factor of four.

Figs. 5 and 6, the higher density simulation in Fig. 10 has achieved substantially less amplification prior to saturation $\left(a_{2} \simeq 0.15\right.$ compared to $\left.a_{2} \simeq 0.25\right)$ than the lower density simulation of Fig. 7 despite what appears to be initially stronger depletion of the pump. Again, though more energy is backscattered from the pump at higher densities, this energy only contributes to forming the secondary oscillations of the $\pi$-pulse and not to amplifying the leading spike, while the more rapid development of disruptive instabilities terminates the amplification process sooner than in the lower density case.

The $k_{x}$ spectrum from this simulation pictured in Fig. 11 shows essentially the same saturation mechanisms and scattered signals (all shifted in $k_{x}$ according to the higher plasma density) as in Fig. 9. Initially, the seed pulse is seen to broaden in $k_{x}$ and amplifies as before. Beginning at $\omega_{1} t=49,400$, however, a much stronger

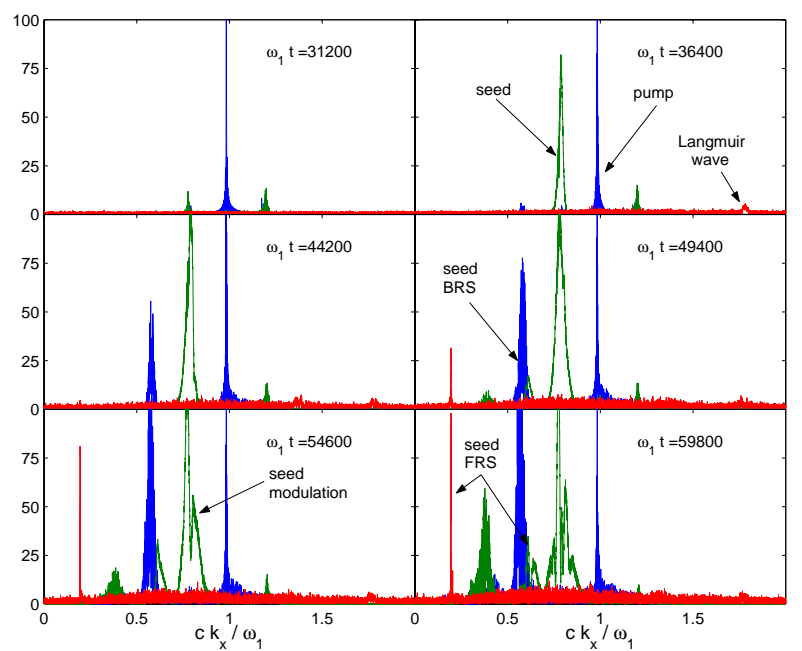

FIG. 11: $k_{x}$ spectrum from the Zohar simulation of Fig. 10. As in Fig. 9, the right-propagating power spectrum is shown in blue, the left-propagating spectrum in green, and the spectrum of the longitudinal electric field is shown in red.

signal corresponding to FRS of the seed is visible, consonant with the higher plasma density. Overall, much higher Langmuir fluctuation levels are evident also consonant with the higher plasma density. Notably, the BRS instability of the seed has grown sufficiently strong at this higher density that secondary BRS of the BRS signal from the seed is clearly distinguishable as the broad right propagating spike down-shifted from the initial seed by $2 \omega_{p e} / c$. The stronger chaotic oscillations in the pump pulse after the passage of the seed are also evidence of the stronger BRS signal from the seed.

\section{CONCLUSIONS}

Several simplifications were made in developing the results presented here. Firstly, backscatter in only one dimension was considered. As noted in Sec. IV, FRS saturation of Raman amplification is dynamically distinct in one dimension (directly forward scattering) as opposed to two or three dimensions (near forward scattering). Within a reasonable computing time (especially for the very long interaction lengths relevant to Raman amplification), two dimensional simulations proved impractical, however. By employing parallel PIC calculations, this constraint may be relaxed, so that two dimensional saturation (and wave-breaking) can more easily be studied. Secondly, finite temperature modifications to the details of wave-breaking were considered only for the single representative temperature of $T_{e}=200 \mathrm{eV}$. In a realistic experimental setting, the plasma may well be formed at a relatively cold temperature $(\sim 10 \mathrm{eV}$ and subsequently be heated throughout the amplification process by inverse Bremsstrahlung of the pump. While PIC codes rigorously describe many of the details of the plasma re- 
sponse, inverse Bremsstrahlung heating of the plasma is typically not accurately modeled due to the smoothing of the microscopic electric fields as a result of the finite spatial grid scale. Hence, a closer investigation of the effect of a time-dependent plasma temperature on wavebreaking (as well as the Raman resonance condition) during amplification is desirable.

In summary, PIC simulations with the Zohar code were used to investigate the effects of wave breaking and saturation in Raman amplification. It was verified that operating as close as possible to the wave breaking limit achieves the strongest amplification of the seed: Once below the density threshold at which the Langmuir wave breaks before the first peak of the seed pulse, the degree of pump depletion and hence seed amplification declines rapidly with decreasing density. For densities above the breaking threshold, stronger depletion of the pump occurs but serves only to amplify the secondary oscillations of the $\pi$-pulse without amplifying the leading spike, while deleterious instabilities which limit the total amplification develop more rapidly and reduce the maximum achievable amplification. In the presence of a representative finite plasma temperature of $T_{e}=200 \mathrm{eV}$, it was found that a plasma density intermediate between the breaking threshold for a cold plasma and the threshold predicted by the theory of Ref. [20] yielded the optimally amplified single spike. At the optimal plasma density, saturation of the amplification effect was observed at an intensity of $\sim 10^{17} \mathrm{~W} / \mathrm{cm}^{2}$ in the form of the amplified pulse breaking into two lobes and depletion of the pump ceasing. Inspecting the $k_{x}$ spectra of these simulations confirmed that Raman forward scattering and modulation instabilities of the amplified seed are responsible for the saturation. Interestingly, and likely due to the exaggerated Langmuir fluctuation levels present in these simulations, substantial backward Raman scattering of the amplified seed was also observed. In all of these simulations, special attention was required to control the unphysically exaggerated noise levels present in the PIC model.

\section{ACKNOWLEDGMENTS}

The help of A. B. Langdon in preparing simulations with Zohar and on the general theory and method of PIC simulation is gratefully acknowledged. This work was supported by the U.S. Department of Energy Contract No. DE-AC02-76-CHO-3073 and the Defense Advanced Research Projects Agency (DARPA).
[1] D. Strickland and G. Mourou, Opt. Commun. 56, 219 (1985).

[2] P. Maine, D. Strickland, P. Bado, M. Pessot, and G. Mourou, IEEE J. Quantum Electron. 24(2), 398 (1988).

[3] V. M. Malkin, G. Shvets, and N. J. Fisch, Phys. Rev. Lett. 82(22), 4448 (1999).

[4] N. J. Fisch and V. M. Malkin, Phys. Plasmas 10(5), 2056 (2003).

[5] T. M. Antonsen, Jr. and P. Mora, Phys. Rev. Lett. 69(15), 2204 (1992).

[6] A. G. Litvak, Sov. Phys. JETP 30(2), 344 (1970).

[7] C. Max, J. Arons, and A. B. Langdon, Phys. Rev. Lett. 33(4), 209 (1974).

[8] G.-Z. Sun, E. Ott, Y. C. Lee, and P. Guzdar, Phys. Fluids 30(2), 526 (1987).

[9] V. M. Malkin, G. Shvets, and N. J. Fisch, Phys. Plasmas 7(5), 2232 (2000).

[10] G. M. Fraiman, N. A. Yampolsky, V. M. Malkin, and N. J. Fisch, Phys. Plasmas 9(8), 3617 (2002).

[11] A. A. Balakin, G. M. Fraiman, N. J. Fisch, and V. M. Malkin (2003), submitted to Phys. Plasmas.

[12] J. P. Verboncoeur, A. B. Langdon, and N. T. Gladd, Computer Phys. Commu. 87, 199 (1995).

[13] H. J. Lee, P. J. Mardahl, G. Penn, and J. S. Wurtele,
IEEE Trans. Plasma Sci. 30(1), 40 (2002).

[14] P. Mardahl, H. J. Lee, G. Penn, et al., Phys. Lett. A 296, 109 (2002).

[15] D. S. Clark and N. J. Fisch, Operating regime for a backward Raman laser amplifier in preformed plasma, to appear, Phys. Plasmas (2003).

[16] A. B. Langdon and B. F. Lasinski, in Methods in Computational Physics, edited by J. Killeen, R. Alder, S. Fernbach, and M. Rotenberg, Advances in Research and Applications (Academic Press, New York, 1976), vol. 16, p. 327.

[17] C. K. Birdsall and A. B. Langdon, Plasma Physics via Computer Simulation (Adam Hilger, New York, 1991).

[18] V. M. Malkin, G. Shvets, and N. J. Fisch, Phys. Rev. Lett. 84(6), 1208 (2000).

[19] V. M. Malkin, Y. A. Tsidulko, and N. J. Fisch, Phys. Rev. Lett. 85(19), 4068 (2000).

[20] T. P. Coffey, Phys. Fluids 14(7), 1402 (1971).

[21] J. N. Dawson, Phys. Rev. 113(2), 383 (1959).

[22] W. B. Mori, C. D. Decker, D. E. Hinkel, and T. Katsouleas, Phys. Rev. Lett. 72(10), 1482 (1994).

[23] A. S. Sakharov and V. I. Kirsanov, Phys. Rev. E 49(4), 3274 (1994). 


\section{External Distribution}

Plasma Research Laboratory, Australian National University, Australia

Professor I.R. Jones, Flinders University, Australia

Professor João Canalle, Instituto de Fisica DEQ/IF - UERJ, Brazil

Mr. Gerson O. Ludwig, Instituto Nacional de Pesquisas, Brazil

Dr. P.H. Sakanaka, Instituto Fisica, Brazil

The Librarian, Culham Laboratory, England

Mrs. S.A. Hutchinson, JET Library, England

Professor M.N. Bussac, Ecole Polytechnique, France

Librarian, Max-Planck-Institut für Plasmaphysik, Germany

Jolan Moldvai, Reports Library, MTA KFKI-ATKI, Hungary

Dr. P. Kaw, Institute for Plasma Research, India

Ms. P.J. Pathak, Librarian, Insitute for Plasma Research, India

Ms. Clelia De Palo, Associazione EURATOM-ENEA, Italy

Dr. G. Grosso, Instituto di Fisica del Plasma, Italy

Librarian, Naka Fusion Research Establishment, JAERI, Japan

Library, Plasma Physics Laboratory, Kyoto University, Japan

Research Information Center, National Institute for Fusion Science, Japan

Dr. O. Mitarai, Kyushu Tokai University, Japan

Dr. Jiangang Li, Institute of Plasma Physics, Chinese Academy of Sciences, People's Republic of China

Professor Yuping Huo, School of Physical Science and Technology, People's Republic of China

Library, Academia Sinica, Institute of Plasma Physics, People's Republic of China

Librarian, Institute of Physics, Chinese Academy of Sciences, People's Republic of China

Dr. S. Mirnov, TRINITI, Troitsk, Russian Federation, Russia

Dr. V.S. Strelkov, Kurchatov Institute, Russian Federation, Russia

Professor Peter Lukac, Katedra Fyziky Plazmy MFF UK, Mlynska dolina F-2, Komenskeho Univerzita, SK-842 15 Bratislava, Slovakia

Dr. G.S. Lee, Korea Basic Science Institute, South Korea

Institute for Plasma Research, University of Maryland, USA

Librarian, Fusion Energy Division, Oak Ridge National Laboratory, USA

Librarian, Institute of Fusion Studies, University of Texas, USA

Librarian, Magnetic Fusion Program, Lawrence Livermore National Laboratory, USA

Library, General Atomics, USA

Plasma Physics Group, Fusion Energy Research Program, University of California at San Diego, USA

Plasma Physics Library, Columbia University, USA

Alkesh Punjabi, Center for Fusion Research and Training, Hampton University, USA

Dr. W.M. Stacey, Fusion Research Center, Georgia Institute of Technology, USA

Dr. John Willis, U.S. Department of Energy, Office of Fusion Energy Sciences, USA

Mr. Paul H. Wright, Indianapolis, Indiana, USA 
The Princeton Plasma Physics Laboratory is operated by Princeton University under contract with the U.S. Department of Energy.

\author{
Information Services \\ Princeton Plasma Physics Laboratory \\ P.O. Box 451 \\ Princeton, NJ 08543
}

Phone: 609-243-2750

Fax: 609-243-2751

e-mail: pppl_info@pppl.gov

Internet Address: http://www.pppl.gov 